\title{
ON THE RECIPROCAL RELATIONSHIP BETWEEN LIFE HISTORIES AND POPULATION DYNAMICS
}

\author{
ODO DIEKMANN \\ $C W I$ \\ P.O. Box 4079 \\ 1009 AB Amsterdam, The Netherlands \\ and \\ Institute for Evolutionary and Ecological Sciences \\ University of Leiden \\ Kaiserstraat 63 \\ 2311 GP Leiden, The Netherlands \\ and \\ J.A.J. (HANS) METZ \\ Institute for Evolutionary and Ecological Sciences \\ University of Leiden \\ Kaiserstraat 63 \\ 2311 GP Leiden, The Netherlands
}

\section{INTRODUCTION}

A population can be thought of as having, at a particular time, a definite structure. Here "structure" is defined as a frequency distribution of characteristics (here and below we are not referring to relative but to absolute frequencies). Usually the structure changes, both on a population dynamical time scale and over evolutionary time. One of the things we want to understand is how the patterns of change are related to mechanisms. These mechanisms necessarily act on the individual level ( $i$-level). Their reduction to population dynamical essentials amounts to a description of the way in which growth, reproduction, probability of dying and influence on the environment are determined by the state of the individual organism ( $i$-state) and the condition of the environment $(E-$ condition). Here the "environment" is thought to encompass all relevant abiotic factors as well as the local abundances of organisms of the same and other kinds (ranging from food to competitors, predators, and parasites). In this view, the natural representation of density dependence is as a feedback through the environment.

Life histories are subject to variation and in the course of evolutionary time the predominant life history may have undergone substantial development. One of the things we, as scientists, want to understand is how, and to what extent, past population dynamics has determined the currently prevailing life history. The same feedback loop via the environment provides the environmental arena for the race of alternative individual life histories to predominance.

How do individual life histories determine population abundance and why did the prevailing life histories evolve? To gradually come closer to a general answer to such broad questions we need formalisms of sufficient generality, a broad spectrum of techniques and lots of case studies. In this paper we shall concentrate on one particular formalism 
of at least some generality and on some implied techniques. Of course there will be quite a contrast between our wishful thinking and the actual state of affairs. The title of this paper refers to an ambitious program and we hope that the text may stimulate at least some readers to contribute to its realization.

Section 2 is concerned with formalism: what kind of mathematical equations best represent physiologically structured population models? This question is unfortunately less trivial than it may seem at first sight.

In section 3 we emphasize the importance of dynamical systems lab facilities for dealing with this kind of models and we speculate a little about the possibilities for a technical realization.

In section 4 we briefly sketch one formulation of the evolutionary question. Section 5 finally sketches in a somewhat anecdotal fashion the wider perspective against which our present considerations should be judged. The result is a cautious optimism: our formalism may still cover less than we might wish, but there are also good reasons to believe that at least some of the results obtained by that formalism have a considerably wider applicability.

This paper does not review the state-of-the-art, nor does it strive for completeness; it clearly ignores many important and interesting issues. Our primary focus will be some aspects of structured population dynamics we expect to shortly undergo rapid development. We hope that we may in addition provide some pointers to a further future.

\section{WHAT SORT OF EQUATIONS?}

Deterministic mathematical modelling, as opposed to e.g. stochastic simulation, necessarily involves a translation of verbal descriptions of assumptions into mathematical equations. In the finite dimensional case, any continuous time dynamical system is uniquely characterized by a system of ordinary differential equations and consequently here "modelling" amounts to a specification of those differential equations on the basis of submodels for the underlying processes. Physiologically structured population models necessarily bring us into the realm of infinite dimensional dynamical systems. Formally the situation is the same, with the ordinary differential equations replaced by partial differential equations (see Metz \& Diekmann, 1986, Heijmans 1986 ${ }^{\text {a,b }}$, Metz, de Roos \& van den Bosch, 1988, Diekmann, to appear). However, a precise interpretation and justification of the limits involved in deriving the partial differential equations can be a horrendous, if not impossible, task. So the risk that a conscientious mathematical analysis never surmounts this, biologically rather uninteresting, stage is substantial. Repeating some of the formulations of Diekmann, Gyllenberg, Metz \& Thieme, preprint, we shall now indicate an attractive alternative for model formulation which can avoid much, if not all, of the unilluminating technical labour.

Let the $i$-state take values in $\Omega$, a measurable subset of $R^{n}$. At the $i$-level, we introduce two model ingredients, one to describe growth and survival and one to describe reproduction. Each of these may itself be composed of various more elementary building blocks. 
We define

$$
\begin{aligned}
u\left(t, t_{0}, y_{0}\right)(\omega)= & \text { probability that an individual that is in state } y_{0} \text { at time } \\
& t_{0} \text { will still be alive at time } t \text { and have } i \text {-state in the } \\
& \text { (measurable) subset } \omega \text { of } \Omega .
\end{aligned}
$$

where it is implicitly understood that the environmental variables are given as a function of time (a point to which we return below). The interpretation requires that $u$ satisfies the Chapman-Kolmogorov consistency relation

$$
u\left(t+s, t_{0}, y_{0}\right)(\omega)=\int_{\Omega} u(t+s, t, y)(\omega) u\left(t, t_{0}, y_{0}\right)(d y)
$$

Likewise we define the reproduction kernel (cf. Jagers, 1989, 1991, preprint)

$$
\begin{aligned}
\Lambda\left(t, t_{0}, y_{0}\right)(\omega)= & \text { expected total number of children, with state-at-birth } \\
& \text { in } \omega \text { born in the time-interval }\left[t_{0}, t\right] \text { from an individual } \\
& \text { having at time } t_{0} i \text {-state } y_{0}
\end{aligned}
$$

$\Lambda$ should satisfy the additive consistency relation

$$
\Lambda\left(t+s, t_{0}, y_{0}\right)=\Lambda\left(t, t_{0}, y_{0}\right)+\int_{\Omega} \Lambda(t+s, t, y) u\left(t, t_{0}, y_{0}\right)(d y)
$$

In the special case of deterministic growth one postulates the existence of a function $Y\left(t, t_{0}, y_{0}\right)$ giving the $i$-state at time $t$, given that the $i$-state was $y_{0}$ at time $t_{0}$ and given survival. One then takes the survival function $\mathcal{F}$ as a second building-block and puts

$$
u\left(t, t_{0}, y_{0}\right)=\delta_{Y\left(t, t_{0}, y_{0}\right)} \mathcal{F}\left(t, t_{0}, y_{0}\right)
$$

where, as usual, $\delta_{y}$ denotes the unit measure concentrated in the point $y$. Substituting (2.3) into (2.1) we find that $y$ should have the semigroup property

$$
Y\left(t+s, t_{0}, y_{0}\right)=Y\left(t+s, t, Y\left(t, t_{0}, y_{0}\right)\right)
$$

while $\mathcal{F}$ should satisfy the consistency condition

$$
\mathcal{F}\left(t+s, t_{0}, y_{0}\right)=\mathcal{F}\left(t+s, t, Y\left(t, t_{0}, y_{0}\right)\right) \mathcal{F}\left(t, t_{0}, y_{0}\right)
$$

In a priori modelling often both $Y$ and $\mathcal{F}$ will be derived from a differential equation, viz.

$$
\begin{aligned}
\frac{d Y}{d t} & =v(Y, E), \quad Y\left(t_{0}, t_{0}, y_{0}\right)=y_{0}, \\
\frac{d \mathcal{F}}{d t} & =-\mu(Y, E) \mathcal{F}, \quad \mathcal{F}\left(t_{0}, t_{0}, y_{0}\right)=1,
\end{aligned}
$$

where $E$ denotes the contemporary environmental condition. 
Similarly one can express $\Lambda$ by the formula

$$
\Lambda\left(t, t_{0}, y_{0}\right)=\int_{t_{0}}^{t} \int_{\Omega} p(y, E(\tau)) \lambda(y, E(\tau)) u\left(\tau, t_{0}, y_{0}\right)(d y) d \tau
$$

where

$\lambda(y, e)=$ expected rate at which an individual in $i$-state $y$, currently living under environmental condition $e$, gives birth

and

$p(y, e)(\omega)=$ probability that a neonate born from a mother with $i$-state $y$ under environmental condition $e$ has itself $i$-state in the (measurable) subset $\omega$ of $\Omega$

In the special case of deterministic $i$-movement (2.8) reduces to

$$
\Lambda\left(t, t_{0}, y_{0}\right)=\int_{t_{0}}^{t} p\left(Y\left(\tau, t_{0}, y_{0}\right), \mathrm{E}(\tau)\right) \lambda\left(Y\left(\tau, t_{0}, y_{0}\right) \mathrm{E}(\tau)\right) \mathcal{F}\left(\tau, t_{0}, y_{0}\right) d \tau
$$

Such a formula does, however, not necessarily produce an unambiguous result. For instance, when $\lambda$ has discontinuities as a function of $y$, these have to be crossed transversally by $Y$, in the sense that the set $\left\{\tau: \lambda(\cdot, E(\tau))\right.$ is discontinuous at $\left.Y\left(\tau, t_{0}, y_{0}\right)\right\}$ has measure zero, since otherwise the right hand side of (2.11) does not yield a well-defined number. In a partial differential equation involving the ingredients $p, \lambda, \mu$ and $v$ this difficulty is rather hidden and has to be tackled during the analysis. In the present approach the difficulty is considered during the modelling phase, when $\Lambda$ has to be specified. To make the step to the population level ( $p$-level), we simply assume that $\Lambda$ is well-defined and has suitable properties. Note, furthermore, that when we start from data, rather than from model equations, $Y, \mathcal{F}$ and $\Lambda$ are the measured quantities and not the various rates.

We now turn our attention to the $p$-level. The population size and composition at some time $t_{0}$ is described by a (Borel) measure $m$ on $\Omega$. At time $t>t_{0}$ both the size and the composition are changed as a result of growth ( $i$-state change), death and reproduction. Let us call the group of individuals which were already present at time $t_{0}$ the zero'th generation and let us introduce the generation development operators $U_{0}$ by

$$
\left(U_{0}\left(t, t_{0}\right) m\right)(\omega)=\int_{\Omega} u\left(t, t_{0}, y_{0}\right)(\omega) m\left(d y_{0}\right)
$$

The Chapman-Kolmogorov equation (2.1) guarantees that $U_{0}$ is an evolutionary system:

$$
U_{0}\left(t+s, t_{0}\right)=U_{0}(t+s, t) U_{0}\left(t, t_{0}\right)
$$


The expected cumulative number of direct offspring in the time interval $\left[t_{0}, t\right]$, as distributed with respect to the $i$-state at birth, is given by the reproduction operators

$$
\left(K\left(t, t_{0}\right) m\right)(\omega)=\int_{\Omega} \Lambda\left(t, t_{0}, y_{0}\right)(\omega) m\left(d y_{0}\right)
$$

The consistency condition (2.2) yields, when lifted to the $p$-level,

$$
K\left(t+s, t_{0}\right)=K\left(t, t_{0}\right)+K(t+s, t) U_{0}\left(t, t_{0}\right)
$$

which is the defining relation for a "cumulative output family" (a notion which was, in the special case of autonomous problems, introduced by Diekmann, Gyllenberg \& Thieme, to appear).

The total births from all subsequent generations together are obtained by iterating the family $K$, with due care for the time structure. The resulting operators $R\left(t, t_{0}\right)$ we shall indicate as the (accumulated) births operators. So $\left(R\left(t, t_{0}\right) m\right)(\omega)$ is the expected cumulative number of all births with $i$-state at birth in the set $\omega \subset \Omega$, in the time interval $\left[t_{0}, t\right]$, given that the population at time $t_{0}$ was described by the measure $m$. Since any newborn is either the offspring of an individual already present at time $t_{0}$ or of an individual born after time $t_{0}$ we conclude that $R$ and $K$ are related to each other by the Stieltjes renewal equation

$$
R\left(t, t_{0}\right)=K\left(t, t_{0}\right)+\int_{t_{0}}^{t} K(t, \tau) R\left(d \tau, t_{0}\right) .
$$

Solving this equation by successive approximations we find the generation expansion back again. Once $R$ is known, we can introduce the population development operators $U$ by

$$
U\left(t, t_{0}\right)=U_{0}\left(t, t_{0}\right)+\int_{t_{0}}^{t} U_{0}(t, \tau) R\left(d \tau, t_{0}\right)
$$

to convert $i$-state at time $t_{0}$, respectively at birth, to $i$-state at the current time $t$, while accounting for the possibility of death. One can verify that $R$ is a cumulative output family for $U$, i.e.

$$
R\left(t+s, t_{0}\right)=R\left(t, t_{0}\right)+R(t+s, t) U\left(t, t_{0}\right)
$$

and subsequently that $U$ is an evolutionary system (as it should be):

$$
U\left(t+s, t_{0}\right)=U(t+s, t) U\left(t, t_{0}\right) .
$$

The evolutionary system $U$ is the object we are interested in. It tells us how $p$-states at time $t_{0}$ are mapped onto $p$-states at later times. The operator families $U_{0}$ and $K$ are calculated direct from the model ingredients at the $i$-level. Given these, we solve the renewal equation (2.16) by successive approximations to obtain $R$ and then calculate $U$ by (2.17). 
So far we assumed that all the environmental variables were given functions of time (it is this assumption which guaranteed the linearity of our equations). To fix ideas, suppose that substrate concentration $S$ is one of the environmental variables, that substrate dynamics is described by the logistic differential equation in the absence of consumers and that substrate is consumed at a per capita rate $\gamma(y, S)$. Then we have to add the equation

$$
\frac{d S}{d t}=r S\left(1-\frac{S}{K}\right)-\int_{\Omega} \gamma(y, S) n(d y)
$$

to the description of the system, where $n$ is the measure describing the (consumer) population at the relevant time. So we can substitute for $n$ in the equation $U^{E}\left(t, t_{0}\right) m$, where $m$ is the initial condition at time $t_{0}$ and where we now have emphasized the dependence of $U$ on the environmental variables $E$ by incorporationg $E$ as a superindex in our notation. In this manner (2.20) becomes a functional differential equation for $S$, since $E$ involves $S$ as one of its components and $U^{E}\left(t, t_{0}\right)$ thus depends on $S$ as a function on the interval $\left[t_{0}, t\right]$. It remains to solve this functional differential equation (equivalently: it remains to find a fixed point for $S$ taking both the internal dynamics and the feedback via the consumer population into account). For this we need a contraction mapping argument and this in turn requires a Lipschitz estimate for $\left\langle\gamma(\cdot, S), U^{E}\left(t, t_{0}\right) m\right\rangle$ as a function of $S$, as a given (continuous, say) function on $\left[t_{0}, t\right]$. Here special properties of either $\gamma(\cdot, S)$ or $m$ may be helpful or even essential.

A general theory for nonlinear problems involving feedback via the environment will in all probability essentially follow the line of arguments sketched for the particular example above. Lots of technicalities are still to be elaborated in detail. The point of this section is simply to demonstrate that one can define the evolution operators $U^{E}\left(t, t_{0}\right)$ in terms of explicit expressions and an abstract renewal equation, avoiding alltogether functional partial differential equations and their cumbersome interpretation! In other words, we can construct the relevant dynamical systems on the $p$-level, both in the nonautonomous linear case and in the nonlinear case, by formulating the model directly as an integral equation, rather than in terms of infinitesimal generators.

\section{HOW TO ANALYSE THE EQUATIONS?}

Not only are ordinary differential equations convenient for the formulation of models, they are, quite often, much more amenable to analysis than their infinite dimensional analogues. This applies to their quantitative, numerical, analysis as well as to their qualitative analysis. In the two-dimensional case, phase plane analysis is an extremely powerful tool. In higher dimensions one often can resort to perturbation methods and bifurcation theory. The higher the dimension, the more difficult this becomes in practice, and perhaps it is more just to make a distinction between low-dimensional and highdimensional than between finite- and infinite-dimensional.

In recent years, the power of the computer as an aid for the analysis of dynamical systems has quickly transcended the strictly numerical domain. Guckenheimer (1991) gives a nice exposition of the underlying philosophy. Programs for the automatic stability and bifurcation analysis of low dimensional dynamical systems, combined with sophisticated 
graphical output facilities, have created possibilities for a qualitative study of models which would escape most understanding when the tools would be restricted to pencil and paper. The program AUTO of E. Doedel (Doedel, 1986) has had a tremendous success in the area of biological applications. More recent developments are LOCBIF (Khibnik, 1990, Khibnik et al., 1992) and DSTOOLS (Guckenheimer, 1991).

More often than not, biological models are meant to investigate the relation between mechanisms and dynamic behaviour in an idealized context, rather than as a quantitatively accurate description of reality. In such situations, the qualitative viewpoint is essential and therefore the new computer tools are of the utmost importance.

But how can we make all this to bear on the infinite dimensional physiologically structured population models? Let us first emphasize that, in our opinion, it will prove to be essential for the applicability of these models that a users friendly computational environment (including nice graphical output facilities) for the qualitative analysis of these models be developed. A crucial step will be the approximation of the infinite dimensional dynamical systems by finite dimensional ones. In principle, there is a multitude of ways in which this can be done. For the special case of deterministic $i$-movement, de Roos, Diekmann and Metz (1992) developed a method based on the approximation of the measure describing the population size and composition by a sum of finitely many concentrated measures. Biologically, these concentrated measures correspond to cohorts and thus the approximation has the attractive feature that it allows an immediate biological interpretation. For each existing cohort the equations describing the change in numbers due to death and the change in position in $i$-state space $\Omega$ due to growth are obtained direct from the model ingredients. To derive the equations for the "cohorts in creation" (i.e. the cohorts which are gradually formed from newborns) one has to perform a careful analysis, see de Roos, Diekmann \& Metz (1992) and de Roos (1988). A discrete time element is introduced by the, to some extent arbitrary, decision to stop to add newborns to a particular cohort and to start the formation of a new one. It is convenient to renumber the cohorts, in much the same way as in the discretized age Lesley matrix model, at such instants, to keep the range of cohort numbers within bounds and to facilitate the interpretation as a discrete time dynamical system. An efficient interface between the implementation of this technique, called the "escalator boxcar train", as developped by de Roos, and programs like LOCBIF, AUTO and DSTOOLS seems realisable in a period of a couple of years. The main problem is that the approximation may, though finite dimensional, be rather high dimensional. (Therefore it remains of great importance too to enlarge our toolbox for model simplification, i.e. for the derivation of meaningful caricatures; see Greiner, Heesterbeek \& Metz, submitted, Diekmann, Metz \& Sabelis, 1988, Metz \& Diekmann 1986, 1991, Nisbet \& Gurney 1983, 1986, Gurney, Nisbet \& Blythe, 1986, Val \& Metz, preprint).

As a side-remark we want to mention that a treatment of the numerical stability and convergence properties of the escalator boxcar train is still missing (some initial steps have been taken by de Roos \& Metz, 1991). This may be an attractive challenge for numerical analysts interested in weak * convergence. The method as described is the lowest order variant and higher order versions are obtained by looking at higher moments of "local" pieces of the measure (see de Roos, 1988). So one can also view the method as a kind of moving finite element method, where movement is along the characteristics 
of the first order partial differential equation. For the special case of one-dimensional $\Omega$ alternative methods exist, see Banks et al., 1988, Ito et al., 1991, Huyer, preprint. We feel enthusiastic about the escalator boxcar train though, since it (i) works whatever the dimension of the $i$-state space $\Omega$ and (ii) admits a biological interpretation.

We hope that in the future physiologically structured population models will receive more attention from numerical analysts, since, we think, pluriformity is a conditio sine qua non for the advancement of any field of scientific activity. The main message of this section is that the attention should go both to the direct numerical integration and to the automatic stability and bifurcation analysis with graphical output.

\section{AN EVOLUTIONARY PERSPECTIVE; FIRST GO.}

For a start, consider a population living under constant environmental conditions. Will the population grow or decline?

A newborn individual having $i$-state $y_{0}$ at birthtime 0 is expected to produce during its entire life a number of offspring

$$
\Lambda\left(\infty, 0, y_{0}\right)(\omega)
$$

with state-at-birth in $\omega$. In other words, $\Lambda\left(\infty, 0, y_{0}\right)$ is the measure describing the expected total offspring production and the corresponding $i$-states at birth. Next, consider a group of newborn individuals with $i$-state-distribution described by the measure $m$. The assumption of constant environmental conditions implies that $\Lambda\left(t+t_{0}, t_{0}, y_{0}\right)=$ $\Lambda\left(t, 0, y_{0}\right)$ and, in particular, that $\Lambda\left(\infty, t_{0}, y_{0}\right)=\Lambda\left(\infty, 0, y_{0}\right)$. (In words: production of offspring does not depend on the moment of birth of the mother.) So if we consider this group of newborn individuals, their birth times may all be different, yet their expected total lifetime offspring production is

$$
\int_{\Omega} \Lambda(\infty, 0, \eta) m(d \eta)
$$

The operator $G$, mapping measures onto measures, defined by

$$
G m=\int_{\Omega} \Lambda(\infty, 0, \eta) m(d \eta)
$$

is called the next-generation operator. The biological interpretation immediately yields that $\Lambda$ should map positive measures onto positive measures, i.e. $G$ should be a positive operator. So under minor technical conditions (Schaefer, 1974, Krasnosel'skiǔ, et al., 1989) the spectral radius of $G$ is a strictly dominant eigenvalue, which we shall call $R$, and if we iterate we find that asymptotically the sizes of subsequent generations differ by a factor $R$, while the composition stabilizes to the eigenvector corresponding to the eigenvalue $R$. Of course $R$ still depends on the (constant) condition of the environment 
$\hat{E}$. In the special case that $\hat{E}=E_{0}$, the "virgin" environment, we shall use the symbol $R_{0}$.

As a side-remark we want to point out the link between the present set-up and our earlier, slightly different, presentation of the same basic issues [Diekmann, Heesterbeek \& Metz, 1990, Diekmann, to appear, Heesterbeek, 1992]. Whenever $\Lambda$ is absolutely continuous in the sense that $B=B(\tau, \xi, \eta)$ exists such that

$$
\Lambda\left(t, 0, y_{0}\right)(\omega)=\int_{0}^{t} \int_{\omega} B\left(\sigma, \xi, y_{0}\right) d \xi d \sigma
$$

we can restrict the attention to generations described by absolutely continuous measures and define $R$ as the strictly dominant eigenvalue of the operator $K$ defined on $L_{1}(\Omega)$ by

$$
(K \phi)(\xi)=\int_{\Omega}\left(\int_{0}^{\infty} B(\tau, \xi, \eta) d \tau\right) \phi(\eta) d \eta
$$

So $K$ can be considered as the restriction of $G$ to the attracting invariant subspace of absolutely continuous measures.

Clearly the population will grow whenever $R>1$ and decline whenever $R<1$. When there is feedback through the environment such that the population stabilizes to a steady state, the corresponding environmental condition $\hat{E}$ should be such that $R(\hat{E})=1$.

The reproduction kernel $\Lambda$ depends on the life history characteristics of the species concerned. Let these be characterized by a variable taking values in a set $\Sigma$. To pose the invasibility question we need to distinguish between the resident, characterized by $u \in \Sigma$, and the invader, characterized by $v \in \Sigma$. The resident will determine, by feedback, the environmental conditions which the invader has to face. Assume that these are steady conditions (the verification of this assumption will, as a rule, involve many more details of the population dynamic model than are required for an analysis of the invasibility question as such). Then we can discuss

$$
R_{0}\left(\hat{E}_{u}, v\right)
$$

the dominant eigenvalue of the next generation operator corresponding to type $v$ in the environment set by $u$. Necessarily $R_{0}\left(\hat{E}_{u}, u\right)=1$. Hence $u$ is an ESS (evolutionarily stable strategy) if the function $v \mapsto R_{0}\left(\hat{E}_{u}, v\right)$ attains its maximum at $u$. In some special situations $R\left(\hat{E}_{u}, v\right)$ factorizes into the product of a function of $u$ and a function of $v$ i.e.

$$
R\left(\hat{E}_{u}, v\right)=\alpha(u) \tilde{R}_{0}(v)
$$

and in that case an ESS corresponds to a maximum of $\tilde{R}_{0}$. It is this characterization of an ESS which one frequently encounters in the literature, usually without any explicit reference to the underlying factorization assumption.

\footnotetext{
${ }^{1}$ Originally, in the context of age-dependent population dynamics in a constant environment, the index zero referred to the zero'th moment of the net maternity function. There is no equivalent of these moments in the general situation, so gradually the interpretation of the index zero changed to what we present here.
} 
We recall and emphasize the basic but questionable assumption made above of a (globally) stable demographic steady state. Certainly cases were the feedback through the environment leads to periodic or even strange fluctuations of (some of) the environmental variables are of truely biological interest. Moreover, in real biology there always are unpredictable external drivers around, such as the weather. For non-constant environments the dominant eigenvalue should be replaced by the dominant Floquet or Lyapunov exponent (see Metz, Nisbet \& Geritz, 1992, for an exposition of the basic ideas in a discrete time context). The main influence this has at the mathematical end is that analytical tractability becomes even far more exceptional than it already is in the case of a constant environment. So we find one more reason to stress the need of dynamical systems lab facilities. At the biological end, fluctuating environments permit a plethora of interesting, and observed, life history strategies (Tuljapurkar 1990, Ferrière \& Clobert 1992) which radically differ from those predicted for the case of constant environments!

The point of this section is the observation that interesting submodels for growth, death and reproduction will lead to reproduction kernels depending on life history parameters and that one can easily pose evolutionary questions in this framework. Under various special assumptions our formulation reduces to the conventional ones. One advantage of the approach taken above is, that it provides a clear perspective, both of the underlying assumptions relevant to the various specific cases and of the relationships linking the various more special approaches. We also hope (and expect) that the framework presented here will: (i) help in the search for further interesting tractable special cases, and, (ii) provide a clearer ecological framework for the extension of ideas like external genetic stability (Eshel \& Feldman, 1982) and the "street-car theory of evolution" (Hammerstein \& Selten, in press) which should provide the ultimate justification for our evolutionary ecological considerations (compare also Charlesworth, 1980).

\section{A LARGER BIOLOGICAL PERSPECTIVE}

The formalism outlined in the previous sections has a certain elegance, otherwise we would not be that hooked to it. But it cannot be everything there is to the world, as the whole approach is predicated upon the assumption that a deterministic individualbased framework holds water. A heuristic discussion of the circumstances under which this will be the case can be found in Metz \& De Roos (1992). These authors also discuss a number of essential open problems inherent in the approach from the previous sections. Their most important observation is that this approach fails when there are long term or preferential interactions among individuals. Such interactions can be of two kinds: (i) individuals seek each other out in an otherwise well mixed assembly. One example occurs in models dealing with pair formation. Another example is when family members stay together in small colonies or herds. (ii) The spatial structure is such that individuals keep bumping into the same opponents. One example is provided by sessile organisms which are stuck into competing (or coöperating) with their immediate neighbours. Another example are models for local mate competition where local populations, though well mixed, are that small that sons are bound to compete predominantly with their brothers. 
When the collection of groups, be it pairs, family herds or local mate competition arena's, forms a well mixed assemblage, then it is still possible to arrive at a deterministic model formulation by treating the groups, and not the separate organisms, as the basic units of discourse, but the resulting formalisms may differ from the one we have considered in the previous section. However, often even this sleight of hand is denied to us, as is exemplified by the sessile organisms. No well-defined deterministic system results when we let for example the number of cells of a stochastic cellular automaton go to infinity while keeping the contact structure fixed! Only when we at the same time let individuals move faster or interact over larger distances will a good deterministic limit obtain (though this certainly has not been proved yet in some generality: a nice discussion of this sort of limits can be found in Spohn, 1991, and De Masi \& Presutti, 1991).

If we do feel that biological reality conforms to a very local pattern of interactions, in which moreover large numbers of individuals are indirectly coupled in a reticulated fashion, mathematics unfortunately has as yet little to offer in the way of general approaches and theorems (but see Liggett, 1985, Durrett, 1988a,b, Durrett and Levin, preprint, Matsuda et al., 1992, and Wolfram, 1986). Our credo, though, is that, while the reality of local interactions should not be denied, much insight can usually be gained from just assuming global, or as a last resort local, well-mixedness, provided a certain amount of care is excercised in interpreting the resulting conclusions. Some results, like the evolutionary stability of a cannibalistic lifestyle or the evolutionary inevitability of the tragedy of the commons, clearly crucially depend on that special, and in those particular cases therefore often ill founded, modelling approximation. But it is usually relatively easy to see when such is the case. Of course cannibalism pays when we assume that the risk of one's own children being eaten does not differ from the risk of being eaten in the population at large, and of course only the ability to cope with an impoverished environment matters, and not the extent to which one's own actions adduce to that improverishment, when we assume that the burden is shared equally.

In order to substantiate the optimistic view expressed in the last paragraph we shall finish by discussing two examples of useful results of which we have good reason to believe that their validity extends considerably beyond the framework of their origin, first discovered, at least by us, in a deterministic individual-based context.

By appending spatial coördinates to the $i$-state variable to arrive at a combined $\mathrm{h}$ (eterogeneity)-variable we can immediately extend the formalism from the previous sections to spatially distributed populations. From now on we shall assume that the population and environmental equations together are translationally invariant over space. In cases where the environment is spatially homogeneous, we can define $R(\hat{E})$ by first integrating out over space. $R_{0}$ we get by specialising to the virgin environmental condition $E_{0}$. Notice that as $h$-space is no longer compact this $R_{0}$ may differ from the Perron root figuring in the same context in, for example, Jagers (preprint).

We shall now concentrate on the linearized dynamics obtained by setting $E$ everywhere equal to $E_{0}$. If we locally introduce a small population it will after a while start growing exponentially iff $R_{0}>1$. In that case it will moreover start expanding radially at eventually constant rates (see e.g. Radcliff \& Rass 1986, van den Bosch et al., 1990, 
and Mollison 1991). If dispersal is not rotationally symmetric the rates will not be the same in all directions. In fact some of those rates may turn out negative, in which case our population marches of towards infinity. The Perron root serves as an indicator: by definition it is larger than one iff our population inherits the whole plane. Our guess is that these results, if reformulated carefully, extend to the full nonlinear case, independent of whether this case admits a well-defined deterministic limit equation, provided (i) under any other environmental conditions that can be encountered, individuals can only do worse than under $E_{0}$ (in the ordering induced on the cumulative birth kernels by the positive cone), (ii) the influence exerted on the environment by the population stays sufficiently localized, (iii) there is a sufficient range of local population densities in between the densities where demographic stochasticity first becomes negligible and those where the effect of the population on $E$ starts to make itself felt. Assumption (ii) is biologically trivial, (i) i.a. implies that there should be no Allee effect and that individuals should not range wider at higher densities, and (iii) is fulfilled whenever propagules are spread around sufficiently widely.

The propagation of so-called focal plant epidemics clearly satisfies assumptions (i) to (iii). The individuals here are the foci or hot spots, i.e. roughly circular areas with a very high density of infection, which derive from a local dispersion of fungal spores in the canopy. These foci interact by bumping into each other, and reproduce through the relatively rare event of long distance spore dispersal. The mechanism by which foci interact cannot be brought under our general framework from the previous sections. Yet we believe (and know from observations) that the results derived from the linearized deterministic considerations apply.

As our second example we notice that many results on the dynamics of individual traits over evolutionary time, including (i) the eventual reachability of ESSes, either direct or through a sequence of protected polymorphisms of diminishing amplitude, and (ii) the characterization of singular points in trait space where protected polymorphisms of ever increasing amplitude get started, on second thought mainly turn out to depend on the existence of a sufficiently smooth function $S(u, v)$ characterizing the possibility of $v$-mutants to invade into a $u$-population (Geritz, Kisdi, Metz, in prep), independent of whether we identify this function with the reproduction ratio $R\left(\hat{E}_{u}, v\right)$ defined in section 4 , the more general dominant Lyapunov exponent $s_{u}(v)$ advertized by Metz, Nisbet \& Geritz (1992), or some measure of inclusive fitness as explained so beautifully by Taylor (1988a,b, 1989).

Of course we need specific models to arrive at concrete biological predictions, but apparently there also exists an exceedingly simple more abstract framework which unifies many of the special results. To arrive at such more encompassing levels of abstraction it helps having available modelling frameworks suggestive of a considerable biological generality which yet are sufficiently near to mainstream mathematics that the relevant abstractions spring to the fore by the simple elegance of their expression. It is this view of the scientific process which sets us on our present course. We hope that also you, dear reader, got some intelectual enjoyment out of our exertions. 


\section{SUMMARY}

From a mathematical point of view, physiologically structured population models are an underdeveloped branch of the theory of infinite dimensional dynamical systems. We have called attention to four aspects:

(i) A choice has to be made about the kind of equations one extracts from the predominantly verbal arguments about the basic assumptions, and subsequently uses as a starting point for a rigorous mathematical analysis. Though differential equations are easy to formulate (different mechanisms don't interact in infinitesimal time intervals and so end up as separate terms in the equations) they may be hard to interpret rigorously as infinitesimal generators. Integral equations constitute an attractive alternative.

(ii) The ability of physiologically structured population models to increase our understanding of the relation between mechanisms at the $i$-level and phenomena at the $p$-level will depend strongly on the development of dynamical systems lab facilities which are applicable to this class of models.

(iii) Physiologically structured population models are ideally suited for the formulation of evolutionary questions. Apart from the special case of age (see Charlesworth 1980, Yodzis 1989, Caswell 1989, and the references given there) hardly any theory exists at the moment. This will, hopefully, change rapidly in the coming years. Again the development of appropriate software may turn out to be crucial.

(iv) Though there clearly exist numerous practical cases that do not naturally fit into our modelling framework, we believe that its combination of a fair amount of realism and elegance is bound to pay dividend, were it only by suggesting useful further generalizations and abstractions.

\section{REFERENCES}

[1] Banks, H.T., L.W. Botsford, F. Kappel and C. Wang. 1988. Modeling and estimation in size structured population models. In: T.G. Hallam, L.J. Gross and S.A. Levin (eds.) Mathematical Ecology, World Scientific, Singapore, 521-541.

[2] Caswell, H. 1989.Matrix Population Models. Sinauer Sunderland, Massachusetts, USA.

[3] Charlesworth, B. 1980. Evolution in Age Structured Populations. Cambridge University Press, Cambridge, England.

[4] De Masi, A. and E. Presutti. 1992. Mathematical Methods for Hydrodynamic Limits, Springer, Berlin.

[5] De Roos, A.M. 1988. Numerical methods for structured population models: the escalator boxcar train. Num. Meth. PDE 4, 173-195. 
[6] De Roos, A.M., O. Diekmann and J.A.J. Metz. 1992. Studying the dynamics of structured population models: A versatile technique and its application to Daphnia. Amer. Nat. 139, 123-147.

[7] De Roos, A.M. and J.A.J. Metz. 1991. Towards a numerical analysis of the escalator boxcar train. In: J.A. Goldstein, F. Kappel \& W. Schappacher (eds.) Differential Equations with Applications in Biology, Physics, and Engineering, Marcel Dekker, 91-113.

[8] Diekmann, O. 1993. An invitation to structured (meta) population models. In S.A. Levin, T.M. Powell and J.H. Steele (eds.) Patch Dynamics, LNBM 96, 162-175 Springer Verlag, Berlin, Germany.

[9] Diekmann, O., M. Gyllenberg, J.A.J. Metz and H.R. Thieme. 1994. The 'cumulative' formulation of (physiologically) structured population models. In: Ph. Clement \& G.Lumar (eds.) Evolutions Equations, Control Theory and Biomathematics, Marcel Dekker, New York etc. Lect. Notes Pure \& Appl. Math. 155, 145-154

[10] Diekmann, O., Gyllenberg, M. and Thieme H.R. to appear. Perturbing semi-groups by solving Stieltjes renewal equations, Diff. Int. Equ.

[11] Diekmann, O., J.A.P. Heesterbeek and J.A.J. Metz. 1990. On the definition and the computation of the basic reproduction ratio $R_{0}$ in models for infectious diseases in heterogeneous populations, J. Math. Biol. 28, 365-382.

[12] Diekmann, O., J.A.J. Metz and M.W. Sabelis. 1988. Mathematical models of predator-prey-plant interaction in a patchy environment. Experimental and Applied Acarology 5, 319-342.

[13] Doedel, E. 1986. AUTO: Software for continuation and bifurcation problems in ordinary differential equations. Cal. Tech., Pasadena.

[14] Durrett, R. 1988a. Lecture Notes on Particle Systems and Percolation, Wadsworth $\&$ Brooks/Cole, Pacific Grove.

[15] Durrett, R. 1988b. Crabgrass, measles and gypsy moths: an introduction to modern probability. Bull. AMS, 18, 117-143.

[16] Durrett, R. and S.A. Levin.1994. Stochastic spatial models: a user's guide to ecological applications. Philosophical Transactions of the Royal Society of London, Series B 343, 329-350

[17] Eshel, I. and M. Feldman. 1982. On evolutionary genetic stability of the sex ratio. Theor. Pop. Biol. 21, 430-439.

[18] Ferriere, R. and J. Clobert. 1992. Evolutionary stable age at first reproduction in a density-dependent model. J. Theor. Biol. 157, 253-267.

[19]Geritz, S.A.H., É. Kisdi and J.A.J. Metz. In preparation. The adaptive geometry of evolutionary trees. 
[20] Greiner, G., J.A.P. Heesterbeek and J.A.J. Metz. Preprint. A singular perturbation problem for evolution equations and time-scale arguments for structured population models.

[21] Guckenheimer, J. 1991. Computational environments for exploring dynamical systems. Int. J. Bifurcation and Chaos 1, 269-276.

[22] Gurney, W.S.C., R.M. Nisbet and S.P. Blythe. 1986. The systematic formulation of models of stage-structured populations. In: J.A.J. Metz and O. Diekmann (eds.). The Dynamics of Physiologically Structured Populations. Springer, Berlin, 474-494.

[23] Hammerstein, P. and R. Selten. In press. Evolutionary game theory. In: R.J. Aumann \& S. Hart (eds.) Handbook of Game Theory with Economic Applications. North-Holland.

[24] Heesterbeek, J.A.P. 1992. $R_{0}$. Ph. D. Thesis, Leiden University.

[25]Heesterbeek, J.A.P. and J.A.J. Metz. 1993. The saturating contact rate in marriageand epidemic models. J. Math. Biol. 31, 529-539

[26] Heijmans, H.J.A.M. 1986a. Structured populations, linear semigroups and positivity, Math. Z.,191, 599-617.

[27] Heijmans, H.J.A.M. 1986b. Markov semigroups and structured population dynamics. In: Aspects of Positivity in Functional Analysis, R. Nagel, U. Schlotterveck, M.P.H. Wolff (eds.), Elsevier, Amsterdam, 199-208.

[28] Huyer, W. Preprint. A size structured population model with dispersion.

[29] Ito, K., F. Kappel and G. Peichl. 1991. A fully discretized approximation scheme for size-structured population models. SIAM J. Num. Anal. 28, 923-954.

[30]Jagers, P. 1989. The Markov structure of population growth. In: Evolution and Control in Biological Systems,A.B. Kurzhanski \& K. Sigmund (eds.) Kluwer, Dordrecht, 103-114.

[31]Jagers, P. 1991. The growth and stabilization of populations, Statistical Science, 6, 269-283.

[32]Jagers, P. Preprint. The deterministic evolution of general branching populations.

[33] Khibnik, A.I. 1986. Numerical methods in bifurcation analysis of dynamical systems: parameter continuation approach. In: Yu.G. Zarhin and A.D. Bazykin (eds.) Mathematics and Modelling, Center of Biological Research of the USSR Academy of Sciences, Pushchino, 162-197 (in Russian).

[34] Khibnik, A.I., Yu. A. Kuznetsov, V. Levitin and E.V. Nikolaev. 1993. Continuation techniques and interactive software for bifurcation analysis of ODEs and iterated maps. Physica D. 62, 360-371.

[35] Krasnosel'skiī, M.A., Je.A. Lifshits and A.V. Sobolev. 1989. Positive Linear Systems - The Method of Positive Operators - Heldermann, Berlin.

[36] Liggett, T.M. 1985. Interacting Particle Systems. Springer, Berlin. 
[37] Matsuda, H., N. Ogita, A. Sasaki and K. Satō. 1992. Statistical mechanics of population: the lattice Lotka-Volterra model. Progress Theor. Phys. 88, 10351049.

[38] Metz, J.A.J. and A.M. de Roos. 1992. The role of physiologically structured population models within a general individual-based perspective. In: D.L. de Angelis \& L.J. Gross (eds.) Individual-based Models and Approaches in Ecology, Chapman \& Hall New York, 88-111.

[39] Metz, J.A.J., A.M. de Roos and F. van den Bosch. 1988. Population models incorporating physiological structure: A quick survey of the basic concepts and an application to size-structured population dynamics in waterfleas. In: B. Ebenman and L. Perron (eds.). Size-Structured Populations: Ecology and Evolution, Springer, Berlin, 106-124.

[40] Metz, J.A.J. and O. Diekmann (eds.). 1986. Dynamics of Physiologically Structured Populations. Lecture Notes in Biomathematics 68. Springer-Verlag.

[41] Metz, J.A.J. and O. Diekmann. 1991. Exact finite dimensional representations of models for physiologically structured populations. I. The abstract foundations of linear chain trickery. In: J.A. Goldstein, F. Kappel and W. Schappacher (eds.). Differential Equations with Applications in Biology, Physics and Engineering. Lecture Notes in Pure and Applied mathematics 133. Marcel Dekker, New York, 269-289.

[42] Metz, J.A.J., R.M. Nisbet and S.A.H. Geritz. 1992. How should we define 'fitness' for general ecological scenarios? TREE 7, 198-202.

[43] Mollison, D. 1991. Dependence of epidemic and population velocities on basic parameters. Math. Biosc. 107, 255-287.

[44] Nisbet, R.M. and W.S.C. Gurney. 1983. The systematic formulation of population models for insects with dynamically varying instar duration. Theor. Pop. Biol. 23, 114-135.

[45] Nisbet, R.M. and W.S.C. Gurney. 1986. The formulation of age-structure models. In: T.G. Hallam and S.A. Levin (eds.) Mathematical Ecology, Springer, Berlin, 95-115.

[46] Radcliffe, J. and L. Rass. 1986. The asymptotic speed of propagation of the deterministic non-reducible $n$-type epidemic. J. Math. Biol. 23, 341--359.

[47] Rand, D.A., H.B. Wilson and J.M. McGlade. Preprint. Dynamics and evolution: evolutionarily stable attractors, invasion exponents and phenotype dynamics.

[48] Schaefer, H.H. 1974. Banach Lattices and Positive Operators, Springer, Berlin.

[49] Spohn, H. 1991. Large Scale Dynamics of Interacting Particles, Springer, Berlin.

[50] Taylor, P.D. 1988a. Inclusive fitness models with two sexes. Theor. Pop. Biol. 34, 145-168.

[51] Taylor, P.D. 1988b. An inclusive fitness model for dispersal of offspring. J. Theor. Biol. 130, 363-378. 
[52] Taylor, P.D. 1989. Evolutionary stability in one-parameter models under weak selection. Theor. Pop. Biol. 36, 125-143.

[53] Tuljapurkar, S. 1990. Population Dynamics in Variable Environments, Springer, Berlin.

[54] Val, J. and J.A.J. Metz. Preprint. Asymptotic exact finite dimensional representations of models for physiologically structured populations: the concepts of weak and asymptotic linear chain trickery.

[55] Van den Bosch, F., J.A.J. Metz and O. Diekmann. 1990. The velocity of spatial population expansion, J. Math. Biol. 28, 529-556.

[56] Wolfram, S. 1986. Theory and Application of Cellular Automata, World Scientific, Singapore.

[57] Yodzis, P. 1989. Introduction to Theoretical Ecology, Harper \& Row, New York. 